This item was submitted to Loughborough's Research Repository by the author.

Items in Figshare are protected by copyright, with all rights reserved, unless otherwise indicated.

\title{
Emotional gait: effects on humans' perception of humanoid robots
}

PLEASE CITE THE PUBLISHED VERSION

http://dx.doi.org/10.1109/ROMAN.2014.6926263

PUBLISHER

(c) IEEE

VERSION

AM (Accepted Manuscript)

LICENCE

CC BY-NC-ND 4.0

REPOSITORY RECORD

Destephe, Matthieu, Martim Brandao, Tatsuhiro Kishi, Massimiliano Zecca, Kenji Hashimoto, and Atsuo Takanishi. 2019. "Emotional Gait: Effects on Humans' Perception of Humanoid Robots". figshare. https://hdl.handle.net/2134/17594. 


\title{
Emotional Gait: Effects on Humans' Perception of Humanoid Robots
}

\author{
Matthieu Destephe, Martim Brandao, Tatsuhiro Kishi, Massimiliano Zecca, Kenji Hashimoto, \\ Atsuo Takanishi, Member, IEEE
}

\begin{abstract}
Humanoid robots have this formidable advantage to possess a body quite similar in shape to humans. This body grants them, obviously, locomotion but also a medium to express emotions without even needing a face. In this paper we propose to study the effects of emotional gaits from our biped humanoid robot on the subjects' perception of the robot (recognition rate of the emotions, reaction time, anthropomorphism, safety, likeness, etc.). We made the robot walk towards the subjects with different emotional gait patterns. We assessed positive (Happy) and negative (Sad) emotional gait patterns on 26 subjects divided in two groups (whether they were familiar with robots or not). We found that even though the recognition of the different types of patterns does not differ between groups, the reaction time does. We found that emotional gait patterns affect the perception of the robot after being shown to the subjects. The implications of the current results for Human Robot Interaction (HRI) are discussed.
\end{abstract}

\section{INTRODUCTION}

In a world built by humans for humans, having a humanoid body is clearly an advantage be it used to work in factories handling heavy loads or performing dangerous tasks in hazardous locations. Advanced humanoid robots, such as robot companions or nursing home robots would have to interact with people of any age in their daily life and need to be able to adapt their behavior accordingly to their feedback. For humans, our daily social interaction is based on multimodal and sometimes redundant communication channels (language, speech tone, gesture, sound, text, etc.). On a humanoid robot, all those channels are not necessarily available usually due to hardware constraints. For example, ASIMO [1], ATLAS [2], HRP-2 [3] (Fig. 1) do not have any face and are expected to interact with humans to complete various tasks. Thus, humanoid robots should use their body and available communication channels as human-like as possible in order to deliver the correct message to its human partner. Our research focuses on the expression of emotions. Emotions strongly

This work was supported in part by Global COE Program "Global Robot Academia", MEXT, Japan and the Waseda special research fund 2013A-888. It is also partially supported by SolidWorks Japan K.K. and DYDEN Corporation The High-Performance Physical Modeling and Simulation software MapleSim used in our research was provided by Cybernet Systems Co.,Ltd. (Vendor: Waterloo Maple Inc.).

M. Destephe, M. Brandao, T. Kishi are with the Graduate School of Science and Engineering, Waseda University, \#41-304, 17 Kikui-cho, Shinjuku-ku, Tokyo 162-0044, JAPAN (e-mail: contact@takanishi.mech.waseda.ac.jp).

M. Zecca is with the School of Electronic, Electrical and Systems Engineering, Loughborough University, UK.

K. Hashimoto are with the School of Creative Science and Engineering, Faculty of Science and Engineering, Waseda University and Humanoid Robotics Institute, Waseda University, Tokyo, Japan.

A. Takanishi is with the Department of Modern Mechanical Engineering, Waseda University, and Humanoid Robotics Institute, Waseda University, Tokyo, Japan. affect social interaction and the expression of emotions is a part of multimodal communication. With emotional gestures, robots might be able to influence the state of mind or reaction of their human partner. Indeed emotions, especially experienced at the time of the decision, can influence directly or indirectly the decision maker's expectation of the probability or desirability of future consequences of said decision [4]. This is further showed by Berthoz et al. [5] who studied how the emotions play a critical role in the complex process of decision-making. We want to affect the perception of people of our robot by solely using its body. By using body movement and gait, we solve the issue when the face is not visible (which can be too far to distinguish facial features) or simply has no facial expression capability) but also, we can convey emotional messages without having the robot to directly interact with people. We may think about several applications of an emotional gait based on the effects on the human perception: intimidate people for law enforcing robots, calm people during disasters, or simply to ease communication and understanding of the robots' intentions during interaction with human partners.

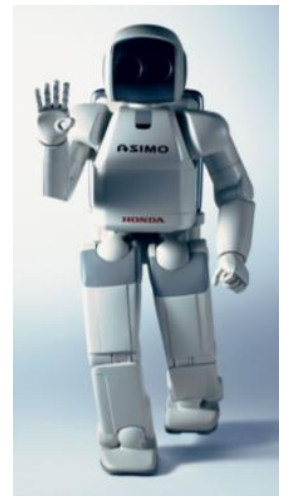

$$
\begin{array}{cc}
\text { a. ASIMO [1] } & \text { (photo: DARPA/Boston } \\
\text { (photo: Honda) } & \text { Dynamics) } \\
\text { Figure 1. Human-sized humanoid robots }
\end{array}
$$

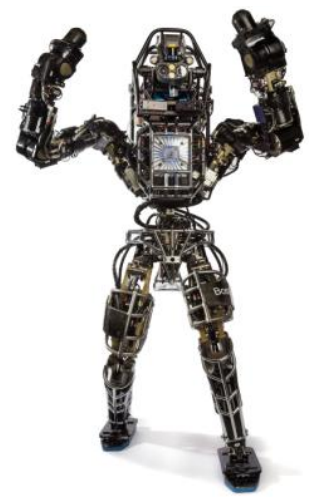

b. ATLAS [2]

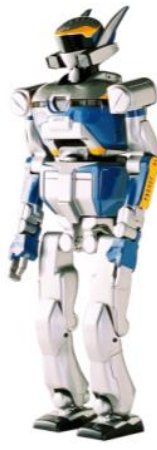

c. HRP-2 [3] (photo: AIST)
Even if it has been shown that one of the most efficient ways of transmitting emotions is to express them with the face [9], emotional body language contains much information used in daily communication interaction between humans [10]. Body language can affect the way facially expressed emotions are perceived and become an important cue to interpret the emotional message [11]. It can also help to distinguish ambiguous facial expressions [12] and increase certainty about the message conveyed to us. Emotion expression through body language can either be an isolated gesture or a different way of moving the body while performing an activity. Recognition of emotions in human motion alone was demonstrated by several studies [13-15]. Another study [16] indicates that the same areas in the brain known for being 
activated by fearful facial expressions are also activated when a fearful body posture is shown with a blurred-out face.

Most works done in Human Robot Interaction (HRI) regarding emotions expression focused on face-based expressions [17-18], emotional speech cues [19] or gestures [20]. There are only few works known to us that used human-sized humanoid robots to express emotions. Lim et al. [21] introduced for the first time emotional walking with the robot WABIAN-RII in 2004. Among other humanoids such as ASIMO or HRP-2, only Geminoid F expresses emotions and only its face is used [22]. However until now, the gait was not used as medium to convey information to people in the robots' surroundings or during direct interaction. As the effects from emotional gait patterns on the users' perception of the robots was never studied, we want to follow this approach and go further by assessing of the effects of an emotional on subjects' perception of the robot.

The two following questions were investigated:

(1) Can the gait of an adult-sized humanoid robot solely express emotions such as happiness or sadness?

(2) Can this emotional gait change the perception that people have of the robot?

With the present paper, we implemented for the first time to our knowledge, biologically-based [23] emotional gait patterns in a human-sized $(148 \mathrm{~cm})$ humanoid robot. We would like to insist on the possible importance of the size of the robot. Hiroi et al. reports that the size of a robot influences the interaction and moreover the threat posed by the robot [24]. They tested three sizes of robot $(60,120$ and $180 \mathrm{~cm})$ and found that a $120 \mathrm{~cm}$-sized robot is the most appropriate for a direct interaction and that robots should be slightly smaller than their human partner.

We will present what we have observed when the robot expressed positive (Happy) and negative (Sad) emotions with its gait. The paper is organized as follows. In section 2, we introduce our robot platform, our approach for emotional pattern generation and the description of the user-study. Section 3 contains the results we obtained. Finally, in section 4 we discuss our findings and conclude with the section 5 .

\section{II.METHOD}

\section{A. Description of the robot}

We conducted our experiments with the humanoid robot WABIAN-2R developed in Takanishi Laboratory at Waseda University (Fig. 2). Unlike most bipedal humanoid robots, WABIAN-2R is able to perform a human-like walking with stretched knees thanks to its 2-DoF waist during the stance phase while other robots walk with bent knees. WABIAN-2R has two 6-DoF legs, 2-DoF waist, 2-DoF trunk, two 7-DoF arms, 3-DoF neck, and two 3-DoF hands [25]. WABIAN-2R has been designed with $1.5 \mathrm{~m}$ in height, and $64 \mathrm{~kg}$ in weight. The kinematic configuration of the structure comprises a total of 39-DoF. Its biomimetical design allows human-like gait including heel-contact and toe-off phases. This robot is mainly used for locomotion experiments and to study human movements. Besides an advanced locomotion technology there are no sensors or other devices that could be used for human robot interaction (HRI), such as a camera or a head capable of emotion expression. Consequently this allows us to concentrate on expressing emotions with body movements only.

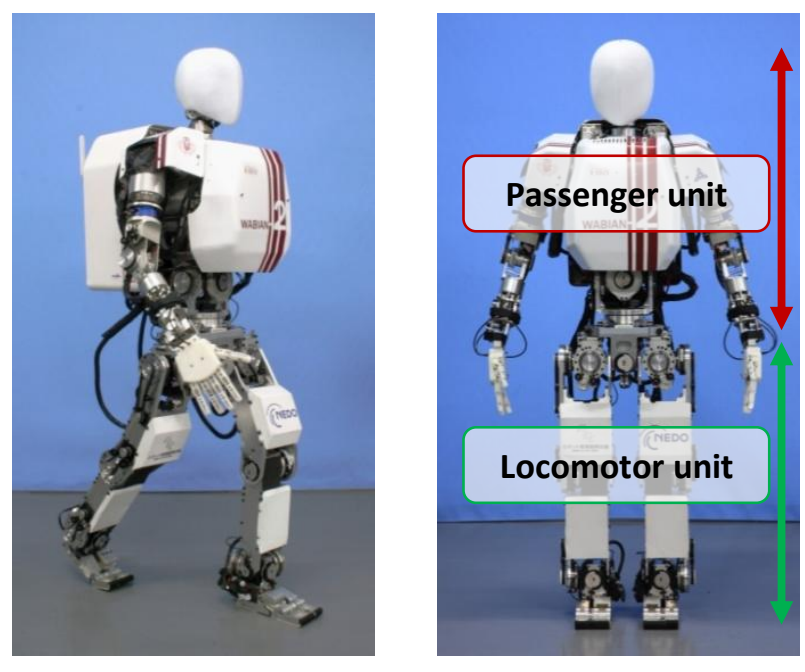

Figure 2. WABIAN-2R platform

\section{B. Description of the patterns}

We asked two professional Japanese actors (who acted in plays, drama and movies) to perform several types of emotional walking such as Sadness, Happiness, Anger and Fear and with different intensities: low, intermediate, high, and exaggerated [23]. We also recorded normal walking.

The humanoid kinematic structure can be divided in two groups: the locomotor unit, formed by both legs and pelvis, and the passenger unit, formed by the head, neck, arms, trunk and also the pelvis. On the locomotor unit gait parameters such as foot height, step length and walking velocity have to be applied. The extracted parameters from the motion recordings cannot be applied directly. Although the human and the robot structures are similar in structure, they are still quite different down to the kinematic properties. Hence the extracted parameters have to be tuned to the robot WABIAN-2R. The extracted emotional walking parameters are normalized in regard to the neutral walking parameter. On the passenger unit the other parameters, the neck pitch, shoulder pitch and the waist pitch, have to be applied. For simplification reasons the pitch of the neck and waist where kept constant during each pattern. We set the shoulder pitch trajectories as close as possible to the extracted parameters within limitations of the robot kinematic structure.

In our previous research, we produced for Happiness and Sadness emotions, three walking patterns of different intensity (intermediate, high and exaggerated) and we created computer graphics videos of our robot WABIAN-2R. For the present study, we extended some of the patterns used in [26] for the virtual robot by improving the stability and doubling the distance walked and we used the real robot instead of the virtual one. We decided to use solely the High intensity category for Happiness and Sadness. We chose this intensity category since it achieved a high recognition rate (Happiness: $76.69 \%$, Sadness: $80.77 \%$ ) when we assessed the patterns in simulation with subjects. We doubled the number of steps for each of the patterns (now 12) and the total walking duration is 
TABLE I. PATTERN PARAMETERS FOR HAPPINESS AND SADNESS

\begin{tabular}{ccccccc}
\hline \hline \multirow{2}{*}{ Parameters } & \multicolumn{2}{c}{ Normal } & \multicolumn{2}{c}{ Happiness } & \multicolumn{2}{c}{ Sadness } \\
& Robot & Actors & Robot & Actors & Robot & Actors \\
\hline $\begin{array}{c}\text { Neck pitch } \\
\text { [degree] }\end{array}$ & 0 & 1.5 & 0 & 2 & 10 & 10 \\
$\begin{array}{c}\text { Shoulder } \\
\text { pitch range } \\
\text { [degree] }\end{array}$ & $-25 / 6$ & $-23 / 10$ & $-35 / 20$ & $-40 / 32$ & $-8 / 8$ & $-8 /+4$ \\
$\begin{array}{c}\text { Waist pitch } \\
\text { [degree] }\end{array}$ & 5 & 17 & 5 & 13 & 10 & 14 \\
$\begin{array}{c}\text { Step height } \\
{[\mathrm{mm}]}\end{array}$ & 32 & 118.3 & 30 & 112.79 & 28 & 86.28 \\
$\begin{array}{c}\text { Step length } \\
\text { [mm] }\end{array}$ & 350 & 592.86 & 370 & 636.15 & 300 & 407.27 \\
$\begin{array}{c}\text { Velocity } \\
{\left[\mathrm{m} \cdot \mathrm{min}^{-1} \text { ] }\right.}\end{array}$ & 23.3 & 62.98 & 30.8 & 108.51 & 17.6 & 36.43 \\
\hline \hline
\end{tabular}

9.63 seconds for Happiness, 12.93 seconds for Sadness and 11.49 seconds for Normal. We also tuned the velocity of the walk to ensure the stability of the robot.

We include the Table I for reference the value of the parameters used for the emotional walking patterns.

\section{Description of the experiment}

In order to assess the effects of emotional gait on the subjects' perception of the robot, we decided to take three parameters into consideration: the emotion recognition rate, the emotion recognition reaction time and the overall feelings towards the robot described by the Godspeed questionnaire [27].

A total of 26 subjects participated in this experiment $(\mathrm{N}=$ $26)$ with an average age of $25.17(\mathrm{SD}=5.9)$ ranging from 20 to 38 years old. All gave written informed consents for participation. We controlled the possible influence of the participants expertise or familiarity with robots. Thus, the participants were divided into two different groups of thirteen people each: the Familiar with robots group $\left(\mathrm{N}_{\mathrm{f}}=13\right)$ and Non-familiar with robots group $\left(\mathrm{N}_{\mathrm{nf}}=13\right)$. The Familiar group subjects were recruited among the researchers and students who were or are currently working with the robot. The Non-familiar group participants, who did not have any previous contact with robots and do not have a peculiar interest in robotics, were invited to participate to a study about Human-Robot Interaction through announcement in class, social network services and mailing-lists. All the participants were briefed about how the experiment will be conducted: six walking patterns will be shown, and for each

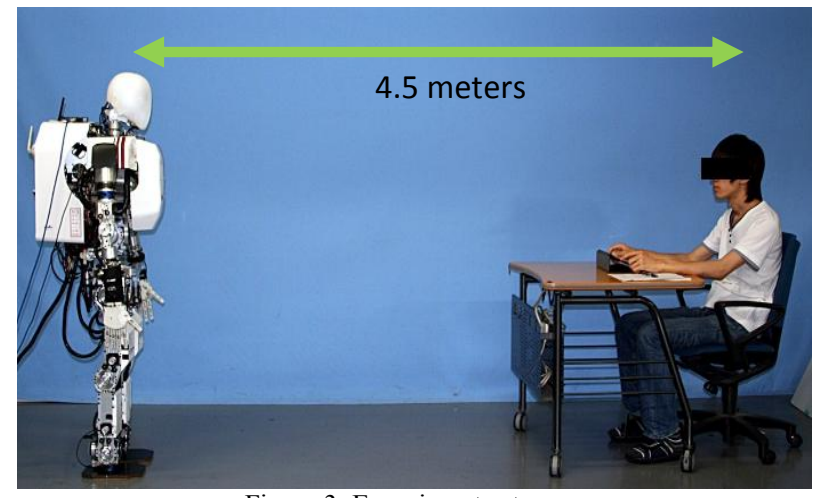

Figure 3. Experiment set up pattern, the emotion recognition answer and the subject impressions were recorded. Subjects were not told that reaction time would be recorded, but they were told to answer as fast as they could.

The robot was standing at 4.5 meters from the subject (Fig.3). The subject was seated at a desk on which a pen, a questionnaire form and a tablet PC were set. The tablet PC was used to record the reaction time of the recognized emotion. The subjects were told to press the button corresponding to the emotion displayed as soon as they thought recognizing it. Six choices were proposed in English and in Japanese: happy, sad, normal, fear, anger and other emotion. At the beginning of the experiment, we showed once to the subjects the normal walking pattern. It was done to serve as a point of comparison with the walking patterns shown after. Each subject saw a total of six walking patterns (Happy x 2, Sadness x 2, Normal x 2) and the patterns were shown in random order. Using a questionnaire, we recorded the age, the time of the experiment and an identification code which identifies the group distinction and differentiates subjects belonging to the same group. We used the Godspeed questionnaire [25] to evaluate how people perceived the robot according to five HRI concepts: anthropomorphism, animacy, likeability, perceived intelligence, and perceived safety. It uses for each concept several semantic variables graded from 1 to 5. The Anthropomorphism concept describes the attribution of human-like features and behavior to non-human things (variables: naturalness, consciousness, life, elegant movements, etc. The Animacy represents the concept of being alive (variables: alive, lively, organic, lifelike, interactive, and responsive). The Likeability depicts the positive impression about others people might have (variables: like, friendly, kind, pleasant, nice). The Perceived Intelligence represents the expected capabilities the robot has (variables: competent, knowledgeable, responsible, intelligent and sensible). The Perceived Safety illustrates the comfort level the people might have with the robot (variables: relaxed, calm and quiescent). A free space was also available for any comment the subjects may have. They were given time to discuss with the experimenters in case they wanted to give oral feedbacks.

\section{RESULTS}

All the parameters (emotion recognition rate, emotion recognition reaction time, Godspeed questionnaire) were analyzed through a two-way ANOVA with one within-subjects factor (emotion \{Happy, Sad, Normal\}) and one between-subjects factor (familiarity with the robots \{Familiar, Non-Familiar\}). Partial $\eta^{2}$ are reported as a measure of the effect size.

\section{A. Emotion recognition}

The results of the emotion recognition rate are presented in the Fig. 4.

The familiarity with the robots did not influence the recognition rate of the emotion expressed in the robot's gait. As expected, the emotions factor has a large statistical 
significant effect on the emotion recognition $(\mathrm{F}(2,48)=$ $10.1071, p<.001, \eta^{2}=0.2$ (large effect)).

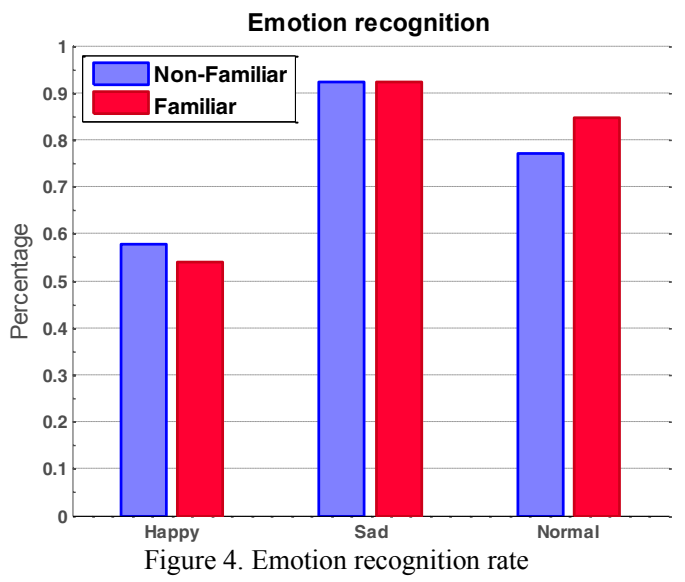

\section{B. Reaction time}

The results of the emotion recognition reaction time are presented in the Table II. The main large effect on the reaction time is due to the Emotions factor $(\mathrm{F}(2,48)=18.0984$, $p<.0001, \eta^{2}=0.16$ (large effect)). Even though there is no statistical difference $(p>0.05)$ between the two groups, the Familiar group reacts faster (14\% faster) to the emotional stimuli than the Non-familiar group. For both groups, the Sad pattern was recognized the fastest and Normal the slowest.

\begin{tabular}{c|c|c}
\multirow{2}{*}{ TABLE II. EMOTION RECOGNITION REACTION TIME (SECONDS) } \\
\cline { 2 - 3 } & \multicolumn{2}{|c}{ Time (in s) } \\
\hline Happy & Non-familiar & Familiar \\
\hline Sad & $13.14 \pm 3.49$ & $11.26 \pm 3.25$ \\
\hline Normal & $10.71 \pm 4.17$ & $9.65 \pm 3.5$ \\
\hline \hline
\end{tabular}

\section{Subjects' Perception of the robot}

First we tested the whole questionnaire results for reliability: Anthropomorphism (Cronbach's $\alpha$ : 0.81), Animacy (Cronbach's $\alpha$ : 0.82), Likeability (Cronbach's $\alpha$ : 0.84 ), Perceived Intelligence (Cronbach's $\alpha$ : 0.81), Perceived Safety (Cronbach's $\alpha$ : 0.68). According to those values, the questionnaire has a good reliability.

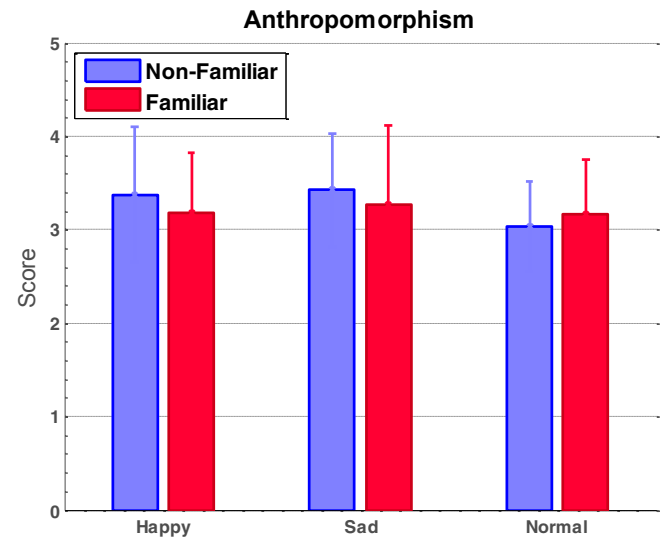

Figure 5. Anthropomorphism score
Anthropomorphism The two-factor ANOVA shows a small significant effect from the Emotion factor $(\mathrm{F}(2,48)=$ $\left.3.2226, p<.05, \eta^{2}=0.03\right)$. The Non-familiar group rated the robot more anthropomorphic for the Happy and Sad patterns than the Familiar group and vice-versa for the Normal pattern. Both groups rated the Sad pattern the most anthropomorphic (Fig.6).

Animacy The analysis does not shown any statistical effect. Nonetheless we can notice that the Non-familiar group rated more and with a very small variance $(\mathrm{M}=3.27, \mathrm{SD}=0.0003)$ than the Familiar group $(\mathrm{M}=2.99, \mathrm{SD}=0.0334)$ (Fig.7).

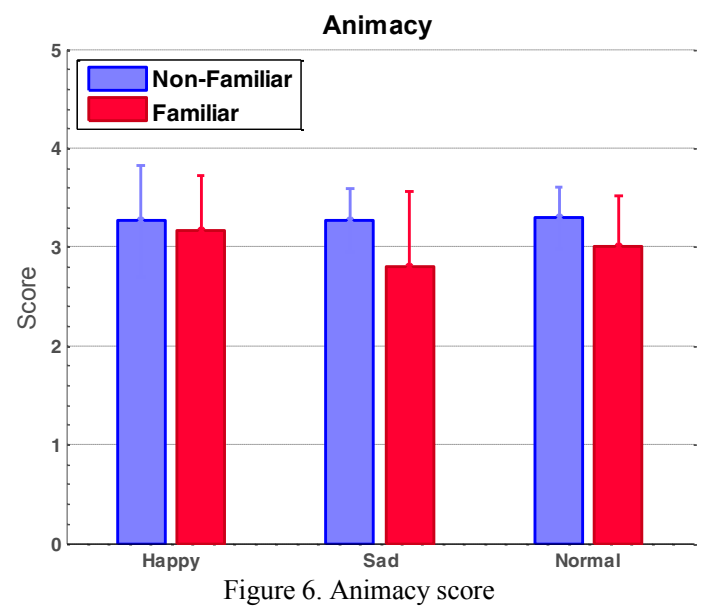

Likeability The analysis showed statistical significance for the two factors: a moderate effect from the Familiarity factor $\left(\mathrm{F}(1,24)=4.6806, p<.05, \eta^{2}=0.11\right)$ and a small effect from the Emotions factor $\left(\mathrm{F}(2,48)=6.713, p<.005, \eta^{2}=0.06\right)$. The Non-familiar subjects rated the robot generally more likeable than the Familiar subjects and both groups rated the Normal pattern the most likeable (close with Happy pattern) (Fig.8).

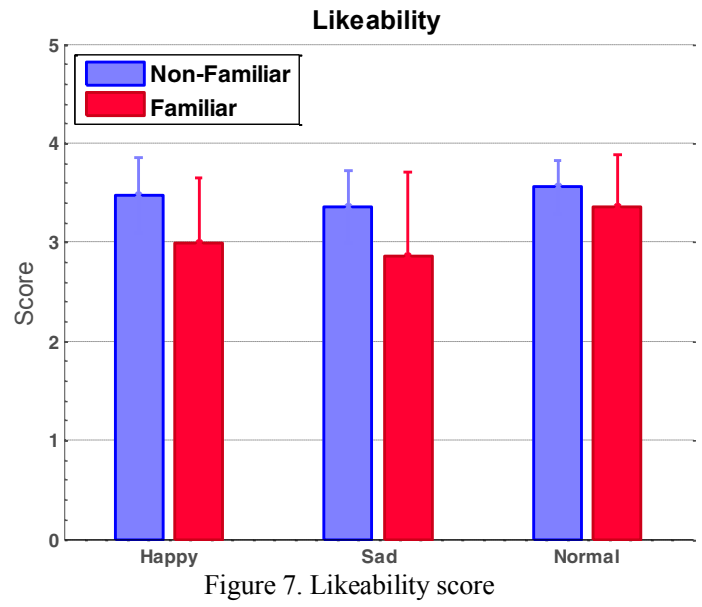

Perceived Intelligence A statistical significance difference with a moderate effect was found for the Emotions $\mathrm{x}$ Familiarity interaction $\left(\mathrm{F}(2,52)=3.466578, p<.05, \eta^{2}=\right.$ 0.04). The Familiar group judges the Happy and Normal pattern equally intelligent and finds the Sad pattern the least intelligent unlike the Non-familiar group (Fig.9). 


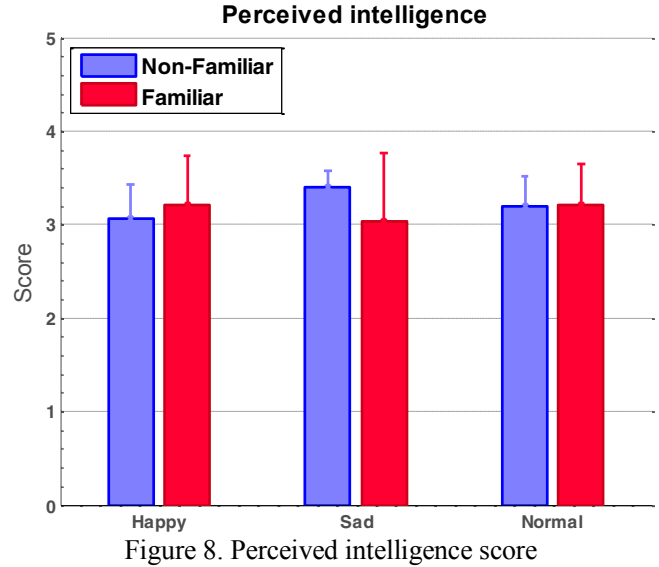

Perceived Safety The analyzed shows a statistical significance difference with a moderate effect from the Emotions factor $\left(\mathrm{F}(2,48)=8.4629, p<.001, \eta^{2}=0.06\right)$. The Non-familiar group rated the Sad pattern the safest and the Happy pattern the least safe. The Familiar group rated equally safe Normal and Happy patterns but less safe the Sad pattern (Fig.10).

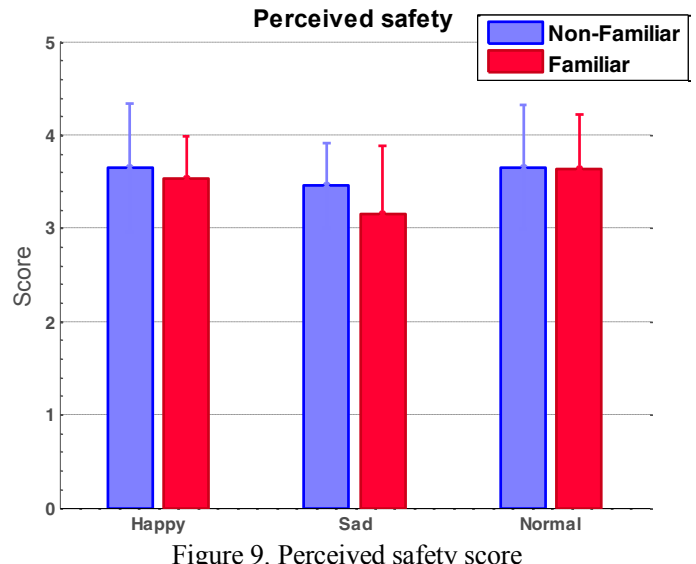

\section{Comments}

We received several comments from the subjects of the two groups with a large majority coming from the Non-familiar group. The subjects from the Familiar group essentially commented in a very practical way on the different moving parts of the robot (step length, arm rotation or parts vibration) and did a few comments on how they think the patterns might be improved. The comments from Non-familiar group were quite different and focused on their feelings. This is supported by the findings in the Animacy part of the questionnaire. The Non-familiar group found the robot more alive and the Familiar group more machine-like. The following comment: "Sometimes the emotion was difficult to understand or to differentiate from one another and I wish the robot had a face to help me decide" was given several times. Regarding the Happy gait demonstration, besides "the robot seems happy" comments, a subject wrote that he "sensed playfulness", another stated that "the robot seems to be happy with a sense of pride and it feels it might look down on others". For the
Sad gait, we had comments such as "I felt that the robot is sad and walked with a broken heart", "the robot is a bit scary and looks like an evil robot from a movie".

\section{DISCUSSION}

First, we wanted to investigate if the gait of an adult-sized humanoid robot solely could express emotions such as happiness or sadness. We achieved an emotion recognition rate of $92.3 \%$ for the Sad gait, 55.8\% for the Happy gait and an overall pattern emotion recognition of $76.3 \%$. Gross et al. report a recognition rate for human emotional walking of 93.3\% for Sad and $66.7 \%$ for Joy [28] and which are similar to what we found while assessing our emotional patterns. This suggests that a robot body does not affect much the recognition of an emotional gait. Moreover, the familiarity with robots did not change the emotion recognition rate. Comparing with our previous research with videos of a virtual WABIAN-2R [26], sadness in the gait of the real robot $(92.3 \%)$ was better recognized than with the 3D modeled robot $(78.2 \%)$ but Happiness recognition $(55.8 \%)$ decreased compared to the virtual robot $(65.38 \%)$. This might be explained by the fact that the patterns were adapted due to the constraint of safety for the robot and to ensure the stability of the walk. The passenger unit parameters were not modified and only the locomotor unit parameters were adjusted to the constraints (velocity, step height and step length). Regarding the results we obtained, the locomotor unit parameters are therefore important for the expression of Happiness in the walk but do not influence much the expression of Sadness. Researchers studied the effects of a positive or negative facial emotion on the recognition speed [29-30]. By using either facial expression pictures or International Affective Picture System set of pictures are shown to the subjects, they found that positive facial expression are recognized faster than negative emotions, unlike our results where we find that negative (Sad) pattern was recognized faster than the positive (Happy) pattern. Face pictures might have a more important and condensed emotional load than a live demonstration and this might explain the reaction time difference between [29-30] and ours. We might also suppose that depending on the type of emotional stimuli (face expression or emotional gait), the recognition pathways are different and then affect the recognition time. One less satisfying point was the recognition of the Happy pattern. Even though the recognition rate of Happiness in the gait by humans is also low $(66.7 \%)$, we should improve the expression of Happiness by, for example, providing context in our future experiments.

Finally, we investigated how the positive (Happy) and negative (Sad) emotional walking patterns influence the perception of the robot. The results showed that the perception was not influenced greatly by the manipulation of the group and was more influenced by the manipulation of the different emotions (or absence of). Among the latent variables of the Godspeed questionnaire, only the Likeability had the Familiarity as one of the main effect $(p<.05)$. All the Godspeed variables besides the Animacy are affected to some degree by the manipulation of the Emotions factor. The emotions affected the perception of the subjects regardless of their experience with robots. As the subjects rated the robot rather positively (scores $>2.5$ ), this means they see the robot 
under a favorable light and seem to not have concerns about it expressing emotions. Nonetheless this should be further investigated to get a deeper understanding of this issue.

\section{V.CONCLUSION}

The current research describes for the first time a adult-size humanoid robot expressing emotion with its gait. It shows that the familiarity with robots seldom influences, if at all, the recognition of the emotion of the robot emotional gait This preliminary research will help use to design interaction scenario with the robot. From the results, we validate our methodology for the design of emotional gait patterns. We are confident that a similar methodology can be applied with success to adult-sized humanoid robots such as ASIMO, ATLAS or HRP-2. This study was conducted without any context and we would like to see how the different emotions expressed by the gait might influence the outcome of the interaction. For example, the gait is quite influential for human predators when they choose their victims and this could be extended to robots [6-7]. This result could be extended to humanoid robots in order to protect them against vandalism [8] by appearing less defenseless when they will wander cities. We would like also to see the difference in the perception when the robot exaggerates its movements and when it expresses Anger. This might give a further insight on the human acceptance of emotional robots in our society.

\section{ACKNOWLEDGMENT}

This study was conducted as part of the Research Institute for Science and Engineering, Waseda University, and as part of the humanoid project at the Humanoid Robotics Institute, Waseda University.

\section{REFERENCES}

[1] Y. Sakagami, R. Watanabe, C. Aoyama, S. Matsunaga, N. Higaki, K. Fujimura, "The intelligent ASIMO: system overview and integration," Intelligent Robots and Systems, IEEE/RSJ International Conference on, vol.3, pp.2478-2483, 2002.

[2] http://www.bostondynamics.com/robot_Atlas.html

[3] K. Kaneko, F. Kanehiro, S. Kajita, H. Hirukawa, T. Kawasaki, M. Hirata, K. Akachi, T. Isozumi, T., "Humanoid robot HRP-2," 2004. Proceedings of ICRA 2004 IEEE International Conference on Robotics and Automation, vol.2, pp.1083-1090, 2004

[4] G. Loewenstein, J.S. Lerner, "The role of affect in decision making". In R. Davidson, K. Scherer, \& H. Goldsmith (Eds.), Handbook of affective science, 619-642, 2003.

[5] A. Berthoz, G. Weiss, Emotion and Reason: The Cognitive Neuroscience of Decision-Making. New York: Oxford Univ. Press, 2006.

[6] A. Book, K. Costello, J.A. Camilleri. "Psychopathy and Victim Selection The Use of Gait as a Cue to Vulnerability." Journal of interpersonal violence 28.11 (2013): 2368-2383.

[7] S. Wheeler, A. Book, K. Costello, "Psychopathic traits and perceptions of victim vulnerability". Criminal Justice and Behavior, 36, 635-648, 2009.

[8] P. Salvini, G. Ciaravella, W. Yu, G. Ferri, A. Manzi, B. Mazzolai, C. Laschi, S-R. Oh, P. Dario, "How safe are service robots in urban environments? Bullying a robot," RO-MAN, 2010 IEEE , pp.1-7, 13-15 Sept. 2010

[9] P. Ekman, W.V. Friesen, "Constants across cultures in the face and emotion", Journal of Personality and Social Psychology, 17, 124-129, 1971.
[10] B. deGelder, "Towards the neurobiology of emotional body language", Nat. Rev. Neurosci., 7(3), 242-249, 2006.

[11] J. Van den Stock, R. Righart, B. de Gelder, "Body expressions influence recognition of emotions in the face and voice", Emotion, 7(3), 487-494, 2007.

[12] H. Aviezer, Y. Trope, A. Todorov, "Body Cues, Not Facial Expressions, Discriminate Between Intense Positive and Negative Emotions", Science, 338(6111), 1225-1229, 2012.

[13] G. Johansson, "Visual perception of biological motion and a model for its analysis", Perception \& Psychophysics, 14, 201-211, 1973.

[14] A. Camurri, I. Lagerlof, G. Volpe, "Recognizing emotion from dance movement : comparison of spectator recognition and automated techniques", Int. J. Human-Computer Studies, 59 (9), 213-225, 2003.

[15] W. Dittrich, T. Troscianko, S. Lea, D. Morgan, "Perception of emotion from dynamic point-light displays represented in dance", Perception, 25(6), $727-7386,1996$.

[16] N. Hadjikhani, B. de Gelder, "Seeing fearful body expressions activates the fusiform cortex and amygdala", Curr. Biol. 13, 2201-2205, 2003.

[17] S. Sosnowski, A. Bittermann, K. Kuhnlenz, M. Buss, "Design and Evaluation of Emotion-Display EDDIE", IEEE/RSJ International Conference on Intelligent Robots and Systems, 3113-3118, 2006.

[18] T. Kishi, T. Otani, N. Endo, P. Kryczka, K. Hashimoto, K. Nakata, A. Takanishi, "Development of expressive robotic head for bipedal humanoid robot", 2012 IEEE/RSJ International Conference on Intelligent Robots and Systems (IROS), 4584-4589, 2012.

[19] T. Ariyoshi, K. Nakadai, H. Tsujino, "Effect of facial colors on humanoids in emotion recognition using speech", 13th IEEE International Symposium on Robot and Human Interactive Communication, 59-64, 2004.

[20] L.Q. Anh, C. Pelachaud, "Expressive gesture model for humanoid robot", ACII'11 Proceedings of the 4th international conference on Affective computing and intelligent interaction - Volume Part II, 224-231, 2011.

[21] H. Lim, A. Ishii, A. Takanishi, "Emotion-based biped walking. Robotica. 22, 5, 577-586, 2004.

[22] C. Becker-Asano, H. Ishiguro, "Evaluating facial displays of emotion for the android robot Geminoid F", 2011 IEEE Workshop on Affective Computational Intelligence (WACI), 1-8, 2011.

[23] M. Destephe, T. Maruyama, M. Zecca, K. Hashimoto, A. Takanishi, "The Influences of Emotional Intensity for Happiness and Sadness on Walking", 35th Annual International Conference of the IEEE Engineering in Medicine and Biology Society (EMBS 2013, 7452-7455, 2013.

[24] Y. Hiroi, A. Ito, "Influence of the Size Factor of a Mobile Robot Moving Toward a Human on Subjective Acceptable Distance", Mobile Robots - Current Trends, 2011.

[25] Y. Ogura, K. Shimomura, H. Kondo, A. Morishima, T. Okubo, S. Momoki, H. Lim, A. Takanishi, "Human-like walking with knee stretched, heel-contact and toe-off motion by a humanoid robot", IEEE/RSJ International Conference on Intelligent Robots and Systems, 3976-3981, 2006.

[26] M. Destephe, M. Zecca, K. Hashimoto, A. Takanishi, "Conveying Emotion Intensity with Bio-inspired Expressive Walking -Experiments with Sadness and Happiness", 22nd IEEE International Symposium on Robot and Human interactive Communication, (RO-MAN 2013), 161-166, 2013.

[27] C. Bartneck, D. Kulić, E. Croft, S. Zoghbi, "Measurement Instruments for the Anthropomorphism, Animacy, Likeability, Perceived Intelligence, and Perceived Safety of Robots", International Journal of Social Robotics. 1, 71-81, 2008.

[28] M. Gross, E. Crane, B. Fredrickson, "Effect of Felt and Recognized Emotions on Body Movements During Walking," in 4th International Conference on The (Non) Expression of Emotions in Health and Disease, pp. 1-2, 2007.

[29] M.G. Beaupré, U. Hess, "An ingroup advantage for confidence in emotion recognition judgments: the moderating effect of familiarity with the expressions of outgroup members", Personality \& social psychology bulletin. 32, 1, 16-26, 2006.

[30] R. Kahana-Kalman, A.S. Walker-Andrews, "The role of person familiarity in young infants' perception of emotional expressions", Child development. 72, 2, 352-69, 2001. 